\title{
A study dealing with primary amenorrhoea with multifactorial etiology - a diagnostic and surgical challenge
}

\author{
Surendra, Vinu Choudhary \\ Correspondence: Dr. Vinu Choudhary, Senior Resident, SMS Medical College, Jaipur, \\ Rajasthan, India; Email - vinuc16@gmail.com
}

Distributed under Attribution-NonCommercial-ShareAlike 4.0 International (CC BY-NC-SA 4.0)

\begin{abstract}
The diagnosis and management of adolescent gynaecological conditions demands the need for dedicated teams to manage this complex group of transitional population between pediatric and adult age group. Primary amenorrhoea is one such condition that can be caused by genetic, endocrine or structural disorders during the development of reproductive organs or may be constitutional in nature. Investigations should be offered in stepwise manner with appropriate counselling. It is important to recognize the spectrum of normalcy before deciding to investigate the abnormal. Treatment depends on the cause with importance on the immediate and long term wellbeing of the individual. We are presenting a case series of primary amenorrhoea depicting multifactorial etiology.
\end{abstract}

Keywords: Primary amenorrhoea, imperforate hymen, MRKH, serous cystadenoma ovary.

In females of reproductive age, with amenorrhea one should first determine whether pregnancy is the etiology. In the absence of pregnancy, it is challenging to determine the exact cause of absent menses ${ }^{1}$. Primary amenorrhea is the failure of menses to occur by the age of 15 years, in the presence of normal growth and secondary sexual characteristics. If by the age of 13 years menses has not occurred and the signs of onset of puberty, such as breast development, is absent, a workup for primary amenorrhea should be started.

Secondary amenorrhea is defined as the cessation of menses after menarche has occurred. Oligomenorrhea is defined as menses occurring at intervals longer than 35 days apart. No consensus has been reached regarding the point at which oligomenorrhea becomes amenorrhea.
Some authors suggest the absence of menses for 6 months constitutes amenorrhea, but the basis for this recommendation is unclear. For a post-menarchal girl or a reproductive-aged woman to experience a menstrual cycle interval of more than 90 days is statistically unusual. Practically speaking, this should be an indication for an evaluation to seek the cause.

Amenorrhea after puberty can be divided into 2 groups: (1) amenorrhea without evidence of associated androgen excess and (2) amenorrhea with evidence of androgen excess (eg, hirsutism, virilization, sexual ambiguity). The relative prevalence of primary amenorrhea (percentages rounded to the nearest tenth) includes hypergonadotropic hypogonadism $(48.5 \%$ of cases), hypogonadotropic hypogonadism (27.8\%), and

Received: $18^{\text {th }}$ July 2018. Accepted: $23^{\text {rd }}$ December 2018.

Surendra, Choudhary V. A study dealing with primary amenorrhoea with multifactorial etiology - a diagnostic and surgical challenge. The New Indian Journal of OBGYN. 2019; 5(2): 150-3 
eugonadism (pubertal delay with normal gonadotropins; $23.7 \%)^{1,2}$.

The hypergonadotropic hypogonadism category includes patients with abnormal sex chromosomes (ie, Turner syndrome), who make up $29.7 \%$ of all primary amenorrhea cases, and those with normal sex chromosomes. The latter group includes both patients who are 46,XX (15.4\%) and those who are 46,XY $(3.4 \%)^{1,3}$. Eugonadism may result from anatomic abnormalities or intersex disorders. Anatomic abnormalities include congenital absence of the uterus and vagina (CAUV; $16.2 \%$ ) or cervical atresia $(0.4 \%)$. Intersex disorders include androgen insensitivity $(1.5 \%)$, 17-ketoreductase deficiency $(0.4 \%)$, and inappropriate feedback $(5.3 \%)^{3}$. No evidence indicates that the prevalence of amenorrhea varies according to national origin or ethnic group. However, local environmental factors related to nutrition and the prevalence of chronic disease undoubtedly have an effect. For instance, the age of the first menses varies by geographic location, as demonstrated by a World Health Organization study comparing 11 countries, which reported a median age of menarche of 13-16 years across centres.

\section{Case 1}

A sixteen year old girl was referred to our gynecologic clinic with ten months history of cyclic abdominal pain, delayed menarche, and lower abdominal swelling. She admitted to have occasional episodes of constipation and urinary retention. Her secondary sexual characteristics were within normal for the age (Tanner stage III). An 18 cms sized suprapubic, tender, cystic, and dull to percussion mass was found on abdominal examination. Perineal examination revealed a bulging pinkish imperforate hymen. Transabdominal ultrasound revealed a grossly dilated uterine cavity, and vagina containing fluid with low level echoes. The kidneys, ureters, uterus and ovaries were normal. The full blood count, serum electrolyte, blood urea and creatinine were also within normal limit. The patient and her mother were counselled on the surgical treatment options and the possibility of loss of virginity during the surgery. They gave consent for the surgery that may preserve her virginity. She had undergone hymenotomy under general anesthesia. Over $800 \mathrm{ml}$ of coffee coloured menstrual blood was evacuated. The urethra was catheterized to avoid its iatrogenic damage. About $2 \mathrm{~cm}$ long cruciate incisions was made on the central portion of the membrane. Minimal trimming of the edges of the hymen was done to prevent defloration. The urethral catheter was removed after the procedure in the theatre. Intravenous augumentin $1.2 \mathrm{~g} 12$ hourly were administered for the first 24 hours, and tablets augumentin $625 \mathrm{mg}$ thrice daily were continued for the next seven days. Her postoperative recovery was uneventful. She was discharged on the third postoperative day, and was to be seen in the gynecological clinic in six weeks.

\section{Case 2}

A $16 \mathrm{yr}$ old girl presented with primary amenorrhoea with lower abdominal pain. On examination slightly tender mass was present in hypogastrium extending to left iliac region. There was a blind vaginal pouch and the secondary sexual characters were normally present. Serum alfa fetoprotein (AFP), serum Ca125 and serum LDH were raised and serum CEA and serum beta HCG were in normal range. Values are $\mathrm{S}$. AFP $=17 \mathrm{IU} / \mathrm{ml}(0.5$ 5.5), $\mathrm{S} . \mathrm{Ca} 125=241.8 \mathrm{IU} / \mathrm{ml}$ (upto 35), S.CEA $=0.9$ $\mathrm{ngm} / \mathrm{ml}$ (upto 2.4) respectively. On endocrinological profile patient was hypothyroid (S. FTSH $=15.3$ $\mathrm{microU} / \mathrm{ml}$, S. FT4 $=.08 \mathrm{ngm} / \mathrm{dl}$, S. FT3 $=1.56 \mathrm{pgm} / \mathrm{dl}$ ). Rest hormonal profile (S. LH, S. FSH, S. Prolactin) were within normal limits. Patient was having bradycardia which was improved after oral thyroxine was started. Ultrasound findings showed absent uterus with a $14 \times 16$ cms right ovarian mass. MRI report showed MRKH syndrome with a $15 \times 17 \mathrm{cms}$ right ovarian hemorrhagic dysgerminoma.

Laparotomy was done where we found a right ovarian cystic mass (around $14 \times 16 \mathrm{~cm}$ ) filled with hemorrhagic fluid with intact capsule. Right sided salpingo oophorectomy was done. Left sided ovary and fallopian tube were normal. There was no extraperitonal involvement of disease. The omentum, lower surface of diaphragm and para aortic lymph nodes were normal. In pelvis, there were two uterinovaginal bulbs connected by a band like uterus, with agenesis of upper vagina. Microscopic examination (histopathological report) of tumour revealed benign serous cyst adenoma of ovary. Cytological examination of peritoneal washing was negative for malignant cells. On karyotyping 46XX chromosomal pattern was found. 


\section{Discussion}

Early diagnosis and timed surgical treatment of an imperforate hymen is important to prevent the complications associated with the delayed treatment after puberty. The diagnosis is usually delayed untill puberty when it presents with its complications like delayed menarche, cyclic lower abdominal pain and a mass, and bulging vaginal membrane. Constipation, urinary retention and infection, pelvic infection and tubo-ovarian abscess, hematosalpix, and endometrosis are the other reported symptomatic presentations of imperforate hymen when treatments were delayed. Our patient also had occasional constipation and retention of urine. A defect on the hymenal barrier can encourage ascending pelvic infection in the accumulated menstrual blood as pelvic inflammatory disease, or as pelvic abscess, and this explained the empirical use of antibiotics in our patient. Menstrual blood had accumulated as hematometra and hematocolpos ${ }^{2,3}$. The timing of surgery is controversial. Some authorities believe that hymenotomy is simple and can be done even in newborns. Virginity is cherished by many religions, cultures and families. Our patient and her mother were counselled about this issue of defloration during surgery. They opted for a virginity-preserving hymenotomy. Minimal edges of the hymen were excised in our patient. Acar et al. $^{4}$ advocated incision and insertion of Foleys catheter as a drain for 14 days for better preservation of the hymen and virginity, but the risk of ascending infection and the discomfort of having insitu Foleys catheter in the young girls made the procedure unpopular. Other consequences or clinical implications that timed hymenotony with good surgical techniques can prevent include recurrence or closure of the opening, scarring of the edges of the hymen and dysparenuia, ascending pelvic infection and infertility, chronic pelvic pain, and ectopic gestation. Most girls who want to conceive after surgery usually achieve pregnancy.

We are presenting a rare case report of MRKH syndrome with hypothyroidism with ovarian tumour (benign serous cyst adenoma) which has not yet been reported in the literature. Diagnosis of the MRKH patients is usually delayed until adolescence. Primary amenorrhea is the reason that the patients wants to visit the clinician. The main feature of this syndrome is the failure of development of the lower end of the Mullerian duct with the normal growth and development of the secondary sexual characteristics. This results in absence of uterus, tubes, cervix uteri and usually the whole vagina. The range of associated abnormalities depends upon the degree of development of the middle and upper thirds of the duct and the Wolfian duct. Approximately one-third of patients with MRKH have renal tract abnormalities, including ectopic kidney, renal agenesis, horseshoe kidney and abnormal collecting tract. Skeletal anomalies are found in 10 to $12 \%$ of cases; spine anomalies are common ${ }^{5-7}$.

Hypothyroidism is another cause of primary amenorrhea. Sexual development and reproductive function is affected by the thyroid hormones. FSH and LH levels do not usually reach to the levels that suffice the ovulation in the patients with hypothyroidism. Myxedema is the clinical presentation of hypothyroidism. But some patients as in our case who appear clinically euthyroid display labaratory evidence of subclinical hypothyroidism $^{8}$.

This patient was given all pertinent information regarding MRKH syndrome as well as treatment options. After this, it was decided to continue regular follow up until she starts having sexual activity. Even with the recent developments in the management of this syndrome, its diagnosis causes significant psychological distress, affecting the patients' quality of life because of the absence of menstruation and impossibility of pregnancy. The distress caused by the diagnosis may be alleviated by surgical or non-surgical treatments, by the passage of time, by counselling, by family's support and by support groups.

\section{Conclusion}

An adolescent female with primary amenorrhoea should be evaluated keeping in mind about its multifactorial etiologies. Proper physical examination and imaging helps in diagnosis of congenital obstructive anomalies and timely surgical intervention is necessary to prevent further complications.

\section{Conflict of interest: None. Disclaimer: Nil.}




\section{References}

1.Junqueira BLP, Allen LM, Spitzer RF, Lucco KL, Babyn PS, Doria AS. Müllerian duct anomalies and mimics in children and adolescents: correlative intraoperative assessment with clinical imaging. Radiographics. 2009; 29(4):1085-103.

2.Muelle GC, Hussain HK, Smith YR. Müllerian duct anomalies: comparison of MRI diagnosis and clinical diagnosis. AJR Am J Roentgenol. 2007; 189 (6): 1294-302.

3.Troiano RN, McCarthy SM. Müllerian duct anomalies: imaging and clinical issues. Radiology. 2004; 233(1):19-34.

4.Acar A, Balci O, Karatayli R, Capar M, Colakoglu MC. The treatment of 65 women with imperforate hymen by a central incision and application of Foley catheter. BJOG. 2007; 114(11): 1376-9

5.Griggs JA, Rudoff J, Coddington CC. M a y e r - R o k i t a $\mathrm{n}$ s k y - K u s t e r - H a u s e r Syndrome with Splenosis. J Reprod Med. 1990; 35-8: 821-3
6.Belver-Pradas J, Cervera-Sanchez J, Boldo-Roda A, Martín-Cortés A, Ferreres-Gómez L. Silver-Russell syndrome associated to Mayer Rokitansky-Kuster-Hauser syndrome, diabetes and hirsutism. Arch Gynecol Obstet. 2001; 265(3):155-7

7.Rhee CS, Kim JS, Woo SK, Suh SJ. MRI of round ligament leiomyoma associated with Mayer-RokitanskyKuster-Hauser syndrome. Abdom Imaging. 1999; 24(2): 202-4

8. Braunwald E, Isselbacher KJ, Petersdorf RG, Wilson JD, Martin JB, Fauci AS. Harrison's Principles of Internal Medicine. In Ingbar SH, ed. Diseases of the Thyroid. Newyork: McGraw-Hill Book Company; 1987:1741-2

\section{Surendra ${ }^{1}$, Vinu Choudhary ${ }^{2}$ \\ ${ }^{1}$ Senior Resident; ${ }^{2}$ Senior Resident, Department of Obstetrics and Gynaecology, SMS Medical College, Jaipur, Rajasthan, India.}

